\title{
Conférence 2
}

\section{L'évaluation}

des médicaments

dans les maladies rares

Loïc Guillevin

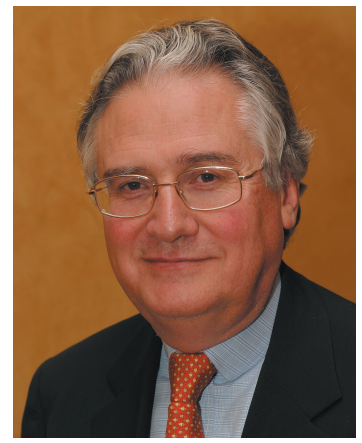

Haute Autorité de Santé et Université Paris Descartes, Hôpital Cochin, AP-HP, France

Le circuit des médicaments et l'AMM

Le circuit du médicament commence par une autorisation européenne donnée par le Committee for medicinal products for human use (CHMP), un comité de l'Agence européenne des médicaments (EMA). II n'y a plus d'AMM demandée uniquement en France. Ensuite, l'autorisation européenne est transmise à l'Agence nationale de sécurité du médicament et des produits de santé (ANSM) qui évalue le rapport bénéfice/risque du médicament, et donne un avis pour une AMM française.

Ensuite intervient la Haute autorité de santé (HAS). Depuis dix ans, la Commission de la transparence attribue deux notes portant, d'une part, sur le service médical rendu (SMR) et, d'autre part, l'amélioration du service médical rendu (ASMR). Elle formule un jugement scientifique sur la qualité du médicament; son prix n'a donc aucune incidence sur la note attribuée.

De son côté, la Commission d'évaluation économique rend un avis d'efficience. Ensuite le Comité économique des produits de santé (CEPS) discute avec le laboratoire du prix du médicament, dans le cadre d'une négociation plus ou moins longue.

Le SMR conditionne le taux de remboursement par la Sécurité sociale ( $65 \%$, $35 \%$ ou $15 \%$ ). Lorsque le SMR est insuffisant, le médicament n'est pas remboursé ; il peut néanmoins être commercialisé.

Les malades en affection de longue durée (ALD) sont remboursés à $100 \%$, quel que soit le taux de remboursement initialement prévu par la Sécurité sociale.

L'ASMR est la seconde note attribuée par la Commission de la transparence. Une ASMR 1 correspond à un médicament innovant, exceptionnel (exemple : un vaccin). Un médicament recevant une ASMR 5 signifie qu'il ne fait pas mieux que son comparateur. Parmi les 650 à 750 médicaments évalués chaque année, un seul reçoit une ASMR 1, quelques-uns reçoivent une ASMR 2 ou 3, et une majorité reçoit une ASMR 5.

L'ASMR influence les discussions sur le prix du médicament. Pour les ASMR de 1 à 3, la base de la négociation s'appuie sur le prix européen. Quand le CEPS (c'est-à-dire le ministère de la Santé) discute du prix, il évolue dans une marge préfixée déterminée par l'avis des autres partenaires. Pour les médicaments ayant reçu une ASMR 4, le prix est négocié et plus élevé que ses comparateurs. Lorsqu'un médicament reçoit une ASMR 5, son prix est obligatoirement moins élevé que celui de ses comparateurs.

La liste en sus, concernant les médicaments prescrits en milieu hospitalier, permet d'avoir un budget spécifique qui s'ajoute au prix de la journée d'hospitalisation. Le médicament ayant reçu une ASMR 1-3 (et parfois 4) peut figurer sur la liste en sus. Un médicament ayant reçu une ASMR 5 ne figure jamais sur la liste en sus, sauf si leur comparateur commercialisé est sur la liste en sus - ce sujet fait l'objet d'une négociation ministérielle.

La liste en sus est établie par le Conseil de l'hospitalisation (qui doit disparaître dans les semaines à venir). Le CEPS y participe, tandis que la HAS n'y participe pas.

Le cheminement d'un médicament dure environ 90 jours, entre le dépôt du dossier et la publication de l'avis définitif.

Les spécificités des médicaments prescrits pour les maladies rares Des règles très particulières ont été mises en place pour les médicaments prescrits pour les maladies rares. Le PHRC et la Fondation maladies rares peuvent, via des programmes de financement nationaux, soutenir le développement d'un certain nombre de molécules pour des 
maladies rares. Diverses exonérations fiscales permettent ainsi aux laboratoires pharmaceutiques d'engager une démarche de développement, tout en limitant leur prise de risque. Des avis précoces sont rendus au niveau européen par l'EuNetHTA (Réseau européen pour l'évaluation des technologies de santé). La HAS participe à ce processus. Un médicament orphelin bénéficie d'une procédure accélérée par la Commission de la transparence. Ainsi, le passage de cette instance est réduit de 90 à 30 jours après le dépôt du dossier. Les dispositions sur les médicaments orphelins sont appliquées au niveau européen. Les exonérations fiscales existantes concernent la taxe sur la promotion des spécialités pharmaceutiques, la taxe payée par l'industrie pharmaceutique, la clause de sauvegarde pour les médicaments orphelins, la taxe sur les ventes directes, et la taxe sur la distribution des médicaments.

Je citerai un exemple sur la clause de sauvegarde. L'Orphacol® (acide cholique) a obtenu une AMM dans les erreurs congénitales de la synthèse d'acides biliaires primaires, dues à un déficit en $3 \beta$-hydroxy- $\Delta 5$ C27-stéroïde-oxydoréductase ou à un déficit en $\Delta 4-3$-oxo stéroïde $5 \beta$-réductase chez les nourrissons, les enfants et les adolescents âgés de 1 mois à 18 ans, ainsi que chez les adultes.

Commercialisé par un autre laboratoire pharmaceutique, le Cholbam ${ }^{\circledR}$ - devenu Kolbam ${ }^{\circledR}$ (acide cholique) - a une indication « restante » car la clause de sauvegarde ne lui permet pas d'aller sur le marché de l'Orphacol® pendant un certain nombre d'années. Pour que le laboratoire qui a conçu la molécule originale obtienne l'AMM et puisse exploiter cette molécule avec un certain bénéfice, aucun autre laboratoire ne peut aller sur ce même marché avec la même molécule et la même indication pendant une période pouvant aller jusqu'à dix ans. Le Cholbam ${ }^{\circledR}$ a donc eu son AMM sur l'indication restante, qui concerne la forme avec les xanthomes tendineux, qui est beaucoup moins fréquente que les autres indications. Quand deux laboratoires conçoivent la même molécule et déposent leur dossier à 8 ou 15 jours d'intervalle, ils bénéficient tous les deux de la clause de sauvegarde.

Le CEPS a réalisé en 2010 une étude sur l'efficience et le coût des médicaments. Ce rapport appelle à la révision des règles conditionnant les médicaments dont le chiffre d'affaires est supérieur à 20 millions d'euros (30 millions d'euros aujourd'hui). Chaque laboratoire propose une somme annoncée globale dépensée pour tous les malades. Si les dépenses sont supérieures, des études médico-économiques beaucoup plus importantes sont menées et peuvent alors quitter le champ des médicaments orphelins.

La loi du 22 mars 2011 permet à des établissements sans but lucratif de devenir des établissements pharmaceutiques pour développer des produits de thérapie génique pour des essais cliniques (exemple du Généthon).

Des règles particulières s'appliquent aux médicaments prescrits pour les maladies rares. Elles portent sur la commercialisation des médicaments aux collectivités, la commercialisation sur la liste en sus (en plus du GHS) et la rétrocession (délivrance du médicament à l'hôpital pour une consommation en ville, selon des règles définies par la Commission de la transparence). Les règles relatives aux listes de prescription pour les médicaments d'exception sont également décidées par la Commission de la transparence, à la demande de la Direction générale de la santé (DGS). Par ailleurs, le PNMRl prévoyait que certains médicaments soient délivrés après accord d'un centre de référence ou de compétence. Cette mesure n'a pas été appliquée. Pour beaucoup de maladies, cela n'a pas eu d'importance, car tous les malades atteints de maladies rares sont suivis dans un centre de référence ou un centre de compétence. Pour un certain nombre de malades, notamment atteints de maladies auto-immunes, la prescription de médicaments n'est toutefois pas encadrée et peut donner lieu à un mésusage ou à une surprescription qui est nuisible à la bonne pratique, et donc à l'utilisation des médicaments.

La France est dotée d'un système de distribution des médicaments assez efficace. Indépendamment des règlements européens sur les médicaments orphelins, les ATU permettent, dès l'obtention de l'AMM, de donner au malade l'accès au médicament - à un tarif non négocié - sans attendre 90 jours. Selon la nouvelle procédure, le laboratoire doit déposer le dossier d'enregistrement dans les six mois suivant le début de l'ATU. Procédure encore plus intéressante, notamment pour les maladies rares, les RTU permettent au patient de bénéficier du médicament hors AMM (ou indication hors AMM).

L'état actuel d'un médicament mis sur le marché s'organise en une étape. Avant la commercialisation, des essais thérapeutiques sont réalisés. Dès sa mise sur le marché, les essais thérapeutiques se poursuivent, mais de manière réduite. De plus, des plans de gestion de risques, des observatoires et des cohortes permettent de surveiller, pendant que l'immense masse de la prescription est opérée.

Une évolution possible, souhaitée par les industriels, consisterait à définir deux étapes. Un médicament concernant très peu de malades - par exemple, une dizaine - peut être considéré comme prometteur. Un SMR et une ASMR n'auraient sans doute pas été donnés au vaccin contre la rage sur le premier malade traité par Pasteur. Or, il arrive que le résultat obtenu suite au traitement d'un premier malade soit mirifique. II ne faut donc pas manquer ce cas. Dans les maladies rares et le cancer, des signaux d'efficacité du médicament sont observés. Une AMM initiale conditionnelle pourrait être accordée à un médicament prometteur. Le laboratoire pourrait ainsi poursuivre ses essais thérapeutiques. $\mathrm{Si}$ les résultats sont confirmés, il obtiendra l'autorisation de remboursement définitive.

Cette évolution reste aujourd'hui une hypothèse. La solution retenue sera peut-être un peu différente, mais la philosophie de l'évolution présentée sera conservée. 


\section{Les enjeux du prix du médicament}

Certains médicaments ne figurant pas sur la liste en sus ont un SMR de 4 ou 5 - ils ne sont donc pas éligibles à la liste en sus, sauf si leur comparateur figure sur cette liste. Ces médicaments se retrouvent dans une situation intermédiaire : ils offrent un bénéfice au malade, mais ce dernier n'est pas guéri pour autant. Ce sujet mériterait une réflexion. Je citerai un exemple relatif au prix de l'innovation thérapeutique. Le Solvadi ${ }^{\circledR}$ traite les hépatites $\mathrm{C}$ avec un taux de guérison de $95 \%$ après trois mois de traitement. Il coûte environ (prix facial) 100000 dollars aux États-Unis, 50000 euros en France, 25000 euros en Grande-Bretagne, 15000 euros en Italie et 700 euros en Égypte ! Le prix est donc fixé de façon arbitraire. Le prix pratiqué en France correspond à celui de la greffe du foie. La variation de prix entre les pays dépend aussi de la taille de la population touchée. Le prix du Solvadi ${ }^{\circledR}$ est peu élevé en Égypte, car le pays compte 20 millions de malades, contre 500000 en France. En outre, il faut savoir que le prix facial n'est pas le prix réellement payé. Le laboratoire cherche toujours à afficher un prix facial élevé.

Un certain nombre de laboratoires réfléchissent à la question de l'efficacité et du financement du médicament en fonction du résultat obtenu. Cette piste devra être explorée. Des accords prix-volume existent déjà : beaucoup d'argent est donné pour peu de malades et peu d'argent est donné s'il y a beaucoup de malades. Un autre principe pourrait être appliqué : si le malade guérit, on paie cher ; s'il ne guérit pas, le remboursement cesse.

On ne peut que se féliciter de molécules efficaces qui prolongent la vie des patients, mais un problème économique reste à régler. L'organisation des soins devrait être revue, notamment en allégeant les structures hospitalières, en développant l'ambulatoire et en mettant en place une surveillance très différente des produits et des malades traités. Ce schéma global s'applique notamment à des molécules en oncologie. Le prix initial fixé pour une faible population de malades a vocation à augmenter de façon continue. Comme ces médicaments sont efficaces, les malades vivent plus longtemps, pour un coût total plus élevé. Si le prix des anciens médicaments reste stable ou diminue en France, il augmente dans certains pays, en particulier aux ÉtatsUnis. Les dépenses consacrées aux maladies auto-immunes représentaient 22,2 \% des dépenses de médicaments de spécialité en 2014, contre $11,3 \%$ en 2006 .

\section{Pourquoi des études post-inscription ?}

La période post-commercialisation fait partie de l'évaluation du médicament. Une fois l'AMM obtenue, le passage en Commission de la transparence effectué, le prix fixé et la commercialisation effective, la large diffusion du médicament à l'ensemble de la population cible peut laisser apparaître des événements insoupçonnés et des effets thérapeutiques non attendus. Cela est particulièrement vrai pour les traitements des maladies rares. Quand un SMR et une ASMR sont donnés sur un essai thérapeutique chez 30 malades avec un suivi de 12 mois pour une maladie qui affectera ces personnes toute leur vie durant, il importe de revoir les produits tous les 5 ans. Des études post-inscription sont alors demandées pour opérer des signaux d'efficacité ou de non-efficacité, ou des effets secondaires. À la fin de l'évaluation, les laboratoires doivent organiser ces études. Nous travaillons en collaboration avec le CEPS qui fixe le prix. L'étude post-commercialisation signifie que la vie d'un médicament ne s'arrête pas le jour où il est dans une boîte sur l'étagère d'une pharmacie.

Les plans de gestion de risques développés au niveau européen portent uniquement sur les effets secondaires. Nous souhaitons pour notre part aller au-delà, avec des confirmations d'efficacité et des réponses apportées à des questions scientifiques qui n'ont pas été abordées dans la première étude ou qui n'ont pas été clarifiées.

Mon dernier transparent porte sur un indicateur intégré fondé sur la comparaison. Une approbation conditionnelle des médicaments pourrait être mise en place. Si un médicament est intéressant pour quelques malades, il pourrait être commercialisé et remboursé. Le laboratoire poursuivrait ensuite son évaluation sur un plus grand nombre de malades pendant une période de 18 mois (par exemple). Si l'efficacité n'est pas au rendez-vous ou si l'effectif de malades n'est pas réuni à l'échéance, la procédure de remboursement disparaîtrait d'elle-même. $\diamond$

\section{ÉCHANGES AVEC LA SALLE}

\section{De la salle}

Qu'en est-il si l'industriel ne parvient pas à réunir suffisamment de patients dans un temps donné?

\section{Loïc Guillevin}

La temporalité, définie de manière contractuelle avec le laboratoire, prend totalement en compte la rareté de la maladie. En Allemagne, le prix fort est accordé immédiatement; si cela ne va pas au bout d'un an, cela s'arrête. Nous procédons différemment en France. Une négociation est menée pour réajuster le niveau de remboursement.

Le dernier transparent de ma présentation est tiré du rapport de Dominique Polton qui a été sollicitée par la ministre de la Santé pour réviser la méthode d'évaluation du médicament. Ce rapport n'a pas encore été remis à la ministre. L'approche proposée suscite beaucoup d'intérêt, notamment de la part des industriels et des malades.

\section{De la salle}

Et quand il n'y a pas de comparateur existant? 


\section{Loïc Guillevin}

Il existe toujours un comparateur ; il s'appelle le soin de support. Nous voudrions évoluer vers une généralisation de l'évaluation comparative.

\section{De la salle}

Le prix des médicaments orphelins est-il corrélé à l'ASMR, l'overall survival et/ou à l'augmentation de la qualité de vie des patients?

\section{Loïc Guillevin}

Le prix des médicaments orphelins n'est pas corrélé à l'ASMR. Comme la Commission de la transparence est en première ligne, elle ne connaît pas le prix du médicament. II se peut que nous évoluions prochainement vers une intégration de la partie médico-économique et de la partie relative à l'évaluation scientifique. Cette évolution présenterait un intérêt, mais aussi des dangers car elle pourrait influencer l'avis d'une commission scientifique.

\section{De la salle}

À quel moment la Commission de la transparence peut-elle recevoir une association lors du processus d'évaluation du service médical rendu?

\section{Loïc Guillevin}

Peu d'associations demandent à rencontrer la HAS. Le service de M. Biosse Duplan s'occupe des relations avec les associations de patients. S'il pense que cela serait utile, une rencontre est organisée. Je reçois parfois des appels téléphoniques ou des courriers. Des médecins m'ont aussi appelé pour me dire que c'était la HAS qui avait écarté tel médicament de la liste en sus. Ce n'est pas vrai. La Commission de la transparence donne un SMR et une ASMR. Si l'ASMR ne satisfait pas le laboratoire, il peut décider de ne pas commercialiser le médicament. Il peut également décider de le commercialiser, mais à un prix et à un taux de remboursement différents de ceux qu'il souhaitait. Quand la HAS dit non, elle donne une ASMR insuffisante - cela n'est jamais le cas pour un médicament qui apporte un bénéfice dans une maladie rare. $\diamond$

\section{LIENS D'INTÉRÊT}

L'auteur déclare n'avoir aucun lien d'intérêt concernant les données publiées dans cet article. 